\title{
Arbor
}

\section{Unamuno y la psicología moderna *}

\section{Rafael Chabrán}

Arbor CLIX, 627 (Marzo 1998), 329-350 pp.

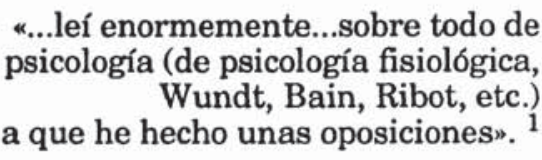

"Mi ambición de momento entones era venir a mi villa natal como catedrático de psicología. ${ }^{2}$

El propósito primordial de nuestro trabajo es trazar la influencia de los seguidores de la psicología fisiológica en el joven Unamuno basándose en un estudio de su "Programa de oposiciones" de 1886 y las lecturas que hizo para prepararse para esas oposiciones y situar esas lecturas dentro de la recepción de la psicología experimental en España, principalmente en las obras de los krauso-positivistas, así como en los alumnos de Salmerón y otros.

\section{Introducción}

Nuestro campo de investigación es el estudio del desarrollo intelectual del joven Unamuno dentro del contexto de la historia de las

* Ponencia leída en las III Jornadas Unamunianas (abril 1996), Salamanca, organizadas por el Servicio de Archivos y Bibliotecas de la Universidad de Salamanca y la Casa-Museo Unamuno. Debo agradecerle a la Profesora D." Carmen Codorñer, Directora del Servicio de Archivos y Bibliotecas y organizadora de las Jornadas por su invitación. Además, es menester celebrar el éxito de D. A Ana Chaguaceda Toledano, Directora de la Casa-Museo Unamuno por la labor excelente que están llevando a cabo al organizar la Casa-Museo para facilitar un mejor acceso y servicio a investigadores. 
ideas que ahora se llama "historia de la mentalidades", "historia de pensamiento español" o aún más específico: "la recepción de las ideas filosóficas y científicas» ${ }^{3}$. Hemos dividido la juventud de Unamuno en tres momentos históricos y tres situaciones geográficas: «Los años universitarios en Madrid (1880-1884)»; «El Regreso a Bilbao (1884-1891) -o sea, los años de las oposiciones-; y finalmente, "Los primeros años salmantinos (1891-1894)" ${ }^{4}$. En el presente ensayo, pensamos concentrarnos en la segunda época, es decir, la época de Bilbao ${ }^{5}$.

\section{El Regreso a Bilbao (1884-1891)}

Después de doctorarse en Madrid en 1884 vuelve a su tierra nativa de Bilbao con su título de doctor en filosofia y letras, lleno de ilusiones "con la resignada aceptación del mandado bíblico de ganarse el pan" ${ }^{6}$ que en España, como sabemos, consiste en preparar y presentar oposiciones. Siete años de preparar y presentar oposiciones; siete años de fracaso. Durante esos años en Bilbao (1884-1891), además de presentar oposiciones, Unamuno da clases particulares; continua con sus investigaciones filológicas y se dedica al periodismo. Después de siete años de fracasar como opositor, Unamuno logró conseguir la cátedra de griego en la vieja universidad salmantina. Entre otras, se prepara y presenta oposiciones para una cátedra de psicología. Durante esta época, Unamuno estudia la nueva psicología fisiológica y experimental. El propósito primordial de nuestro trabajo es trazar la influencia de los seguidores de la psicología fisiológica en el joven Unamuno basándonos en un estudio de su "Programa de oposiciones" de 1886 y las lecturas que hizo para prepararse para esas oposiciones y situar esas lecturas dentro de la recepción de la psicología experimental en España, principalmente en las obras de los krauso-positivistas como en los alumnos de Salmerón y otros.

En su ensayo autobiográfico "Mi mirador de la Cruz", Unamuno relata su preparación para lo que serían cinco oposiciones y nos dice lo siguiente "Preparé lo mejor de mis cinco oposiciones" ${ }^{7}$. $\mathrm{Al}$ leer otros textos nos damos cuenta que en realidad se preparó para ocho oposiciones. Las primeras que preparó fueron las oposiciones para una cátedra de psicología y otra de latín. Él mismo nos dice «...me prepare a hacer oposiciones y las hice primero a una cátedra de psicología, lógica y ética...y después...dos infructuosas oposiciones de latín" ${ }^{8}$.

Su primera oposición, la de "Psicología, lógica y ética", cobró una cierta importancia en su desarrollo intelectual. Como el mismo Unamuno 


\section{Unamuno y la psicología moderna}

nos dice en su ensayo "En mi viejo cuarto" al regresar a Bilbao él ya se veía como profesor de psicología: "Mi ambición de momento entonces era venir a mi villa natal como catedrático de psicología" ${ }^{9}$. Durante los años entre 1884 y 1891, el joven Unamuno dedicaría muchas horas al estudio de la psicología.

Por lo general, los estudiosos y críticos no han prestado la atención adecuada al investigar la importancia que tuvo el estudio de la psicología y el impacto que tuvo ese estudio en el desarrollo del joven Unamuno. Además, es menester situar este interés dentro de la historia de la psicología en España que, como sabemos, forma parte de lo que hoy en día se llama Historia del pensamiento español. Para ver la importancia que cobró el estudio de la psicología moderna en la formación del joven Unamuno sólo tenemos que repasar la famosa y muy citada Carta a Urales. Sobre su evolución intelectual durante los años en Bilbao, Unamuno le escribe a Urales: «...leí enormemente...sobre todo de psicología (de psicología fisiológica, Wundt, Bain, Ribot, etc.) a que he hecho unas oposiciones" ${ }^{10}$. Evidencia de su interés por la psicología se encuentra también, como sabemos, en las muchas citas a psicólogos y temas de la psicología por todo el corpus unamuniano. Sin embargo, sólo tenemos que acercarnos a la biblioteca personal de Unamuno - hoy la Casa Museo- para consultar un número considerable de obras de psicología" ${ }^{11}$.

Unamuno no sólo leía y estudiaba psicología sino que también dictaba clases y daba conferencias sobre temas relacionados con la psicología. En el ya citado ensayo «En mi cuarto» nos dice lo siguiente: "Era yo un muchacho que no llegaba a los veinticinco cuando en un círculo de esta villa tuve a la osadía de dar una conferencia sobre la memoria y su mecanismo" ${ }^{12}$.

La conferencia que Unamuno dictó estaba basada en las obras de T. Ribot y él nos dice cómo había nutrido y fundamentado su conferencia con citas y alusiones a la obra de Ribot, de quien luego hablaremos. Además, también nos cuenta - con poca modestia- algo sobre la recepción de su conferencia. Nos dice que el público quedo asombrado por "cuanto ha leído y cuanto sabe este muchacho" ${ }^{13}$. Sin embargo, a la vez, confiesa que dudaba mucho que alguien del público hubiera aprendido algo de su intervención.

Podemos ubicar las primeras oposiciones que hizo el joven Unamuno - las de 1886 - dentro del contexto de la presentación de esa primera conferencia sobre psicología. Antes de entrar a hablar sobre el contenido y la estructura de esta primera oposición para una cátedra de psicología tenemos que decir algo de qué era y cómo se enseñaba esa materia 
que se llamaba "Psicología, lógica y ética» a finales del siglo pasado. Como sabemos esa materia se daba a nivel de bachillerato y servía como introducción a un curso básico y elemental de filosofia. O sea, el curso de psicología, lógica y ética echaba los cimientos para los cursos de filosofia en la universidad que era lo que se conocía como "Metafísica" en esos tiempos.

\section{El estudio de la psicología en España}

¿Qué se entendía por psicología a finales del siglo pasado? Por supuesto, la psicología oficial de esa época no es la psicología de nuestros días. La psicología de esa época se basaba en el escolasticismo o mejor dicho en el neoescolasticismo que tenía sus raices en el pensamiento de Santo Tomás de Aquino. Es decir, la psicología era fundamentalmente la psychologia rationalis de Santo Tomás: «Psychologia igitur est scientia de anima" ${ }^{14}$. Los libros de texto de psicología de esa época, como los de Ortí y Lara o de Ceferino González, definían la psicología como: el estudio o la ciencia del alma ${ }^{15}$. Para la mayoría de los historiadores de la psicología, o de la filosofía de hoy en día, la psicología de esa época es una psicología sistemática pre-científica en la que las teorías psicológicas son brotes de la epistemología o de la ética. Sin embargo, para los seguidores del pensamiento neo-escolástico, el hombre era principalmente un alma que utilizaba un cuerpo y el estudio de la psicología, lógica y ética tenía como propósito básico el conocer el propósito primordial del hombre, es decir, conocer a Dios y conseguir la salvación. Como sabemos, no fue hasta la ultima parte del siglo XIX -con la llegada del positivismo y otras corrientes del materialismoque la psicología pudo librarse de las especulaciones metafísicas del pensamiento tradicional y neoescolástico. Sólo hasta cuando llegó el momento en que la psicología pudo dejar sus bases idealistas podemos hablar de la existencia de una psicología experimental. En España, como sabemos, esto no ocurrió hasta la llegada del positivismo y el krauso-positivismo.

\section{El joven Unamuno y sus estudios de psicología}

Desde sus primeros cursos de psicología, $\longrightarrow$ sea, desde sus años en el Instituto Bilbaíno- el estudio de la psicología era una de las asignaturas favoritas del joven vasco. En esas clases él aprendió que 


\section{Unamuno y la psicología moderna}

la psicología era el estudio de la naturaleza del animal y del hombre. El alma era la base de la vida y era "psyche» y "mente». A la psicología se le llamaba "ciencia" en la que se estudiaba la mente -o sea, almaen sus muchas funciones y manifestaciones: la conciencia, la sensación y el comportamiento. Los autores de los libros de texto que usaba el joven Unamuno ya conocían algo de las últimas aportaciones de lo que se llamó en esa época la psicología fisiológica, es decir, la base de la psicología moderna. Sin embargo, los autores de esos libros de texto rechazaban la psicología fisiológica porque la consideraban materialista ( $\mathrm{y}$ atea), porque afirmaba la concepción material del alma. Dentro de este contexto podemos entender bien los ataques contra la frenología durante esta época ${ }^{16}$. Ahora bien, claro que existía un grupo de pensadores españoles que conocían bien y bregaban a favor de una psicología "positiva" y experimental. Entre estos pensadores, médicos y científicos, podemos citar nombres como Pedro Mata (1811-1877), Luis Simarro (1851-1921) y otros ${ }^{17}$. Algunos de estos pensadores se habían desarrollado dentro de lo que se ha llamado el krauso-positivismo. Como sabemos, Unamuno tuvo mucho contacto con miembros de este movimiento durante toda su vida. Aunque de una forma muy distinta, podemos situar el afán del joven Unamuno -que viene después- por la psicología fisiológica en los momentos del desarrollo de la psicología krauso-positivista. La psicología "moderna" nace en el seno del krauso-positivismo en los años 70 y 80 del siglo pasado, principalmente en las obras de Urbano González Serrano. Aunque Unamuno tuvo contacto con Francisco Giner de los Ríos y González Serrano, nosotros pensamos que el desarrollo e interés del vasco por la psicología surge de una forma mas bien independiente ${ }^{18}$.

Además, como hemos subrayado en nuestros trabajos sobre la evolución intelectual del joven Unamuno dentro del positivismo, aún después del rechazo del positivismo - después de 1897- Unamuno siempre mantuvo un gran interés por el pensamiento científico y por las ideas evolucionistas como las de Darwin.

Como bien nos podemos imaginar, el contenido de un curso que llevaba como título "Psicología, lógica y ética» sería controlado fuertemente en la España a finales del siglo pasado. No tenemos mas que echarle un vistazo rápido a la Gaceta de Madrid de la fecha de 2 de octubre de 1885 para ver la versión oficial de esa materia. Según este artículo, una parte gruesa del contenido de esta materia versaba sobre los argumentos y las demostraciones sobre la existencia de Dios y las refutaciones e impugnaciones de sistemas filosóficos de índole "materialista». Es preciso recordar que para 1886, el joven Unamuno 
ya había pasado por esos años universitarios en Madrid cuando había intentado "raciocinar" su fe.

«El Programa de psicología, lógica y ética de 1886»

Para prepararse para sus primeras oposiciones, el muy diligente opositor preparó una memoria en un cuaderno que llamó «Programa de psicología, lógica y ética" en abril de $1886^{19}$. Esta obrita o cuaderno, es más bien un esbozo de un temario para lo que luego sería su plan del curso de psicología. En realidad este texto es más bien lo que nosotros llamamos un "Syllabus". Este texto que nosotros consultamos con mucho afán en la Casa Museo hace ya 16 años -ipodría ser?no ha recibido aún el merecido estudio. Aun con el vistazo más rápido, podemos ver que este programa nos dice mucho sobre el desarrollo intelectual del joven Unamuno dentro del pensamiento positivista, el evolucionismo y lo que nos interesa hoy: la psicología fisiológica.

Claro está que el dicho "Programa de psicología, lógica y ética" es más que nada un «borrador» o un "cuaderno de estudio». Al leer este texto nos da la impresión que el joven opositor había confeccionado esta obrita más bien para prepararse para sus exámenes de oposición. Es decir, es una especie de ejercicio o práctica. De ninguna manera debemos tomar este cuaderno como un tratado sistemático y desarrollado sobre la materia. Es nada más que una serie de apuntes organizados para presentar los temas de un curso en cuanto a la materia. Aunque no es una obra elaborada cuidadosamente, sí nos puede ayudar a estudiar la trayectoria del pensamiento y la formación del joven Unamuno y también trazar algo de la historia de la psicología en España durante la última parte del siglo pasado.

Ahora bien, vamos al meollo, al contenido y la estructura del programa. El programa consta de 91 "Lecciones», unas 58 páginas. Con un vistazo sumamente rápido podemos ver que el curso que Unamuno había preparado era totalmente distinto a lo que se daba en las clases de psicología, lógica y ética. Ya desde del principio, veamos, por ejemplo, la "Lección 2", Unamuno empieza su discusión de la psicología como ciencia y su relación con la ciencia experimental. Luego, él pasa a contrastar esa ciencia, la psicología moderna, con la psicología tradicional, o sea, la psicología racional del escolasticismo. En esta sección compara la psicología tradicional con la psicología fisiológica y la psicofísica. 
Desde el principio del programa nos damos cuenta de que Unamuno conocía bastante bien la psicología más avanzada de su tiempo. Nos referimos a la psicología inglesa, francesa, y alemana de la última parte del siglo XIX. En la página 17 del "Programa» encontramos menciones a los pensadores ingleses Alexander Bain (1818-1903) y John Stuart Mill (1806-1873) ${ }^{20}$. De aquí en adelante en el «Programa» vamos a encontrar muchas referencias al positivismo y el pensamiento evolucionista y su relación con la psicología. Se podría decir que uno de los ejes principales del "Programa" se encuentra en la "Lección 40" en la que Unamuno escribe «El hecho como punto de partida». Con esta frase empieza su discusión sobre el positivismo y la coherencia de este punto de vista. Unamuno continúa su discusión sobre el positivismo en la "Lección 43». En esta sección menciona a Herbert Spencer y su obra First Principles, es decir, Primeros Principios. Dicho sea de paso, - y ya lo hemos desarrollado en otro lugar- ésta es una de las primeras referencias de Spencer que hemos encontrado en la obra de Unamuno. Podríamos decir que textualmente éste es uno de los lugares donde podemos ver la influencia de Spencer en el joven Unamuno. Vamos a dejar, por el momento, el contenido del "Programa» de psicología y vamos a pasar a los resultados de esta oposición.

Como bien podemos imaginar la correspondencia de Unamuno puede esclarecer las razones por el fracaso en estas oposiciones. Al hablar de la correspondencia, hablamos por ahora de sus cartas a Pedro Múgica y a Federico Urales. En su carta a Múgica fechada abril 1890, le cuenta a Múgica sobre sus fracasos en las oposiciones que había preparado. No queda duda alguna que se refería a la oposición de psicología de 1886 y a las dos que hizo para una Cátedra de Metafísica (1888-1890). En esa carta, Unamuno escribió lo siguiente: «En psicología no bien hablé de Wundt y entre a explicar algo de lo poco que se relativo a lo mucho que en psicología fisiológica se ha hecho, me tacharon de materialista" ${ }^{21}$.

Bien nos podemos imaginar el choque que recibió el tribunal de oposición al escuchar el nombre de Wundt no cabe duda que las ideas del joven Unamuno en cuanto psicología eran profundamente de índole "materialista", o sea, de acuerdo con la psicología experimental más avanzada de la época.

Muchos años después Unamuno se dio cuenta de que tenía que haber fracasado dada su persona y temperamento. Su espíritu independiente y su sangre juvenil eran, no cabe duda, demasiado para el tribunal. De la misma manera el tribunal no podía aceptar el pa- 
radigma positivista y materialista que proponía Unamuno. En un texto de 1905 Unamuno explica esto muy bien: "Pero dado mi criterio de entonces esa la materia y dado sobre todo mi independencia de juicio que ya por aquella época era mi dote espiritual fracasé y no pude sino fracasar...” ${ }^{22}$.

\section{La historia de la psicología y el krauso-positivismo ${ }^{23}$ en España}

Uno de los primeros que percibe las posibilidades positivistas y sus relaciones con la psicología es el mismo Francisco Giner de los Ríos. Esto se ve claramente en su obra, Lecciones sumarias de Psicología (1874-1877) ${ }^{24}$. En esta obra podemos ver la presencia de la nuevas corrientes de la moderna psicología fisiológica y la psicofísica. En esta obra notamos la fuerte influencia de pensadores como Wundt, Fechner y Helmholtz ${ }^{25}$. A Wundt se le considera el primer psicólogo moderno del siglo XIX. A él se debe el impulso de la psicología experimental. La filosofia de Wundt ha sido calificado con bastante acierto de "filosofia científica». Coincide con Fechner, Lotze y Hartmann en muchos puntos. Las primeras traducciones de Wundt en España se hicieron por Carreras y Sanchis (1880-82) y J. González Blanco (1902). La psicología fisiológica es una psicología como ciencia experimental. Es decir una psicología atenida a los datos proporcionados por la experiencia o por los métodos de las ciencias empíricas. De esta forma, la psicología científica sirve como puente entre la ciencia natural y la filosofía.

La positivización del krausismo se detecta principalmente en el campo de la psicología. Además de la ya mencionada obra de Giner de los Ríos, podemos ver la clara influencia del positivismo en la obra de un krausista salmantino, Mariano Ares, profesor de Metafísica en la Universidad de Salamanca y profesor del ilustre penalista salmantino, Pedro Dorado Montero (1861-1919) que también sintió la atracción por el krausismo y el positivismo ${ }^{26}$. Mariano Arés fue el traductor de la obra de Théodule Armand Ribot (1839-1916) conocida en castellano como La psicología inglesa contemporánea (1877) ${ }^{27}$. En este libro se puede encontrar los logros de la corriente positivista en el campo de la psicología. Este interés por la psicología experimental por parte de algunos krausistas establecerá una base científica para las nuevas concepciones filosóficas basadas en el positivismo. 


\section{Draper en España, «Historia de los conflictos entre Religión y Ciencia», y la psicología fisiológica 28}

La recepción de la psicología fisiológica en España está ligada íntimamente a la obra de Nicolás Salmerón (1836-1908) y la polémica sobre la obra de John William Draper (1811-1829) ${ }^{29}$. Durante su vida Salmerón se encontró en medio de muchas polémicas: polémicas políticas, filosóficas, científicas y religiosas. La ciencia estaba muy presente en las mentes de los españoles durante la Restauración. Esto se puede ver de una forma muy clara si nos acordamos de la importancia de dos hechos sobresalientes de esta época: la polémica sobre la publicación de la obra de Draper y la así llamada polémica sobre «Ciencia Española» ${ }^{30}$, de la que no podemos hablar en estos momentos.

La publicación de la traducción castellana de la obra de John William Draper (Juan Guillermo Draper) ocasionó una de las polémicas ${ }^{31}$ más profundas para Salmerón. Hay que situar la obra de Draper dentro del contexto de la extinción del positivismo y la Mentalidad positiva en España. Se puede decir que la obra de Draper, con su importantísimo "Prólogo" de Salmerón, fue la chispa para la implantación de esa Mentalidad positiva en España. No podemos olvidar que el trasfondo intelectual de toda la polémica de Draper es el krausismo, la libertad de expresión y los momentos de gran efervescencia causadas por la revolución del 68 y la recepción del positivismo y el monismo alemán. Salmerón se encontró en medio de todo este ambiente intelectual.

Es necesario contextualizar la aparación de la traducción de la obra de Draper en España para ver la importancia del papel que jugó Salmerón en esta polémica. La obra de Draper es un precursor de todo un tipo de literatura que surgió entre 1870 y 1900 . Esta literatura esta repleta de «metáforas militares» (conflictos, guerras) que se usan para enfatizar el divorcio entre la religión y la ciencia moderna - el tema fundamental de Draper-.

Dos palabras sobre Draper. Aunque nació en Inglaterra, Draper desarrolló lo grueso de su vida en los Estados Unidos. Este "positivista yankee" fue químico, médico, fisiólogo, filósofo, profesor e historiador. Fue uno de los fundadores de la facultad de la Universidad de Nueva York y aportó mucho al campo de la historia de la fotografía. Pero lo que nos interesa de Draper hoy son sus aportaciones al campo de la historia de las ideas, especialmente a la historia de las ideas filosóficas y científicas.

Antes de entrar al contenido de la obra de Draper y establecer la importancia que tuvo para el pensamiento de Salmerón, tenemos 
que mencionar algo de las influencias que notamos en la obra de Draper. Draper se forma en un ambiente positivista. Más que Herbert Spencer, la influencia que notamos en Draper es la del positivista francés, Augusto Comte. Para Comte, la ley fundamental del progreso y de la historia se encuentra en lo que él denomina la ley de los tres estados. Según Comte, la historia acabará por producir un estado perfecto: el estado positivo. Como ha indicado Diego Nuñez, el pensamiento de Draper está basado en el modelo biologista del progreso y desarrollo. Esta analogía biológica lleva a un historismo determinista.

Ahora bien, vamos la obra de Draper y las polémicas en que participó Salmerón. En 1871, Edwardo Livingston Youmans y Daniel Appleton, los principales divulgadores del positivismo inglés y de las obras científicas del momento en América e Inglaterra, le pidieron a Draper una obra sobre los conflictos entre la religión y la ciencia para La Serie Científica Internacional («The International Scientific Series»). Esta colección tenía como meta editar las obras de los científicos más destacados del momento. Dicho sea de paso, que Youmans fue uno de los más activos propagadores del pensamiento evolucionista a nivel editorial en los Estados Unidos.

La obra de Draper, Historia de los Conflictos de la Religión y la Ciencia se publicó por primera vez en $1874^{32}$. Este libro se convirtió inmediatamente en un «best seller" internacional. Aparecieron traducciones al francés, alemán, italiano, polaco, portugués, holandés, japonés, ruso y por supuesto, al castellano, entre otras lenguas ${ }^{33}$. En España, aparecieron dos traducciones en 1876. Una publicada por la Biblioteca Contemporánea del grupo positivista y neokantiano de José del Perojo. Esta edición se hizo de la versión francesa de la obra de Draper.

La segunda traducción, la que nos interesa más a nosotros, la hizo el astrónomo Augusto T. Arcimís Werhle (1844-1910) de nacionalidad francesa (de origen vasco-francés), miembro de la Sociedad Astronómica de Londres y amigo íntimo de Francisco Giner de los Ríos. Arcimís estaba, por lo tanto, estrechamente vinculado a la Institución Libre de Enseñanza. La traducción de Arcimís se publicó en Madrid en 1876 y se volvió a publicar en 1885, 1886, 1888 y 1903. Además de estas versiones, también aparecieron otras versiones de la obra de Draper en las editoriales de Sempere, Prometeo y Maucci de Valencia así como la Editorial Popular Iberia de Barcelona.

Notamos la presencia del positivismo en el pensamiento de Salmerón a partir de 1875 y esto corresponde perfectamente a las pautas del desarrollo del positivismo tal como nos las presenta Nuñez. En el "Apéndice" a la obra de C. Tiberghien se nos dice que los lazos entre 


\section{Unamuno y la psicología moderna}

la filosofia se han de encontrar en: «un concierto racional entre la ciencia y la filosofia» ${ }^{34}$. Tanto en el "Prólogo" a la obra de Draper, como en el "Prólogo" al libro de H. Giner de los Ríos, Salmerón apunta rotundamente a la psicología físiológica y a la psicofísica como herramientas para enlazar la filosofia y la ciencia. Describe las relaciones entre la filosofía y la ciencia en términos de "un gran concierto":

«El punto de cita, sí vale decir, en que se prepara ese gran concierto, es el cerebro del hombre. De aquí el inmenso interés, y la decisiva trascendencia que ofrece al presente la psicología fisiológica. Ella puede, en rigor ser considerada como la prenda de unión entre las dos tendencias en que se ha dividido hasta ahora la construcción científica" ${ }^{35}$.

Es decir, para Salmerón la razón y la experiencia son los elementos básicos y constitutivos de todo saber científico. Falta un elemento clave en el pensamiento salmeroniano: el monismo.

Veremos la importancia que tuvo el monismo alemán en dos momentos de la vida de Salmerón. En primer lugar, en el importantísimo y ya citado "Prólogo" a la obra de H. Giner de los Ríos, Filosofía y Arte $y$ en la circunstancia de la publicación de la versión castellana de la obra de Haeckel, Morfología general de los organismos (1887) hecha por Salvador Sanpere y Míquel ${ }^{36}$. Sanpere y Míquel tomó la decisión de hacer esta traducción después de una conversación con Salmerón en París.

En el "Prólogo" a Filosofía y Arte, como hemos visto claramente, Salmerón abandona el krausismo para dedicarse partidario militante de un monismo científico. Intenta determinar las relaciones entre la filosofia y la ciencia:

"No basta, hay sobretodo, la especulación para el filósofo, ni puede limitarse a sistematizar los datos de la conciencia; necesita conocer a lo menos los capitales resultados de la observación y la experimentación en la ciencias naturales...la alianza definitiva de la especulación y la expresión...." ${ }^{37}$.

Salmerón, como los otros krausistas cree en la unidad de la realidad y en la necesidad y utilidad de indagar en la composición de la Psicofísica para encontar la unidad indivisa de la realidad. Expresó esta idea, como vemos en el "Prólogo" a Filosofía y Arte y anteriormente en el "Prólogo» a la traducción del libro de Draper:

"Atestiguan en lo general nuestro aserto las graduales evoluciones del llamado protestantismo liberal y la transformación que en el Positivismo contemporáneo prepara el Monismo tan preclaramente representado por Haeckel y Wundt” 38 . 
Para Salmerón todas las concepciones dualistas "se han gastado". A partir de 1878, vamos a ver que Salmerón no tiene más opción que huir de todo tipo de dualismo. Para él, sólo existe «una concepción monista de la realidad» ${ }^{39}$ y la forma de acercarse a esta realidad es por medio de la psicología fisiológica y a la psicofísica.

\section{Nicolás Salmerón, Urbano González Serrano y La psicología experimental en Espana}

La psicología experimental entra en España por medio de los positivistas (Simarro) y los krauso-positivistas, la mayoría de ellos alumnos de Salmerón. Ya hemos visto cómo Salmerón cita a Wundt, Fechner, Lotze, Helmholtz y Spencer. Estos pensadores son muy importantes para la introducción de la psicología fisiológica y la psicofísica en España. Fechner médico, fisiólogo y físico inventó la psicofísica y su obra mas importante fue Elemente der Psychophysick (1860), es decir, Elementos de Psicofísica. Wundt fue el que fundó el primer laboratorio de psicología experimental y su obra clave es Grundzüge der physiologischen Psychologie. (1893), [Prinicipios de Psicologíá fisiológica]. Los discípulos de Salmerón que divulgaron las ideas de la psicología experimental en España fueron Urbano González Serrano, Eusebio Ruiz Chamarro, José Verdes Montenegro y Montoro y José de Caso ${ }^{40}$ entre otros.

Influyó en muchos profesores de filosofia especialmente en sus alumnos pero nadie representa mejor su ideología que Urbano González Serrano (1848-1904), extremeño, nacido en Navalmoral de la Mata. Aunque vivió muchos años en Madrid, siempre se mantuvo en conexión con sus paisanos, especialmente los de Cáceres. González Serrano entregado a la lectura y en constante relación con sus maestros, principalmente con el que fue su iniciador en filosofia, Salmerón. A los 23 años había obtenido el doctorado en Filosofia y Letras y el bachiller en Derecho. Por sus méritos fue nombrado sustituto de Salmerón en la Cátedra de Metafísica. Frecuentaba el Ateneo de Madrid, donde se dió a conocer en los debates principalmente como defensor de las doctrinas científicas mas recientes. Según Valentí Camp:

«González Serrano figuró entre los krausistas y colaboró con su maestro en la traducción y anotación de las obras de Thibergien; pero antes que sus compañeros, y al igual que Salés y Ferre, mostró sus simpatías por el positivismo y tuvo una sincera admiración por Spencer, y más tarde por Lange, Wundt, Fouillee, Fechner, Lotze..." ${ }^{41}$. 


\section{Unamuno y la psicología moderna}

González Serrano siempre fue un pensador de criterio elevado y uno de los pocos publicistas que, teniendo una personalidad vigorosa, consiguieron sustrearse a la inflexibilidad que ha caracterizado casi por igual a los propugnadores españoles de todos los sistemas filosóficos ${ }^{42}$.

La obra de González Serrano también tiene importancia, no sólo porque es un ejemplo de los ardores del desarrollo de la psicología moderna en España, sino por el valor que le da a la psicología en cuanto a la renovación de la educación y la pedagogía. Aquí podemos enlazar la obra y pensamiento de González Serrano con las reformas pedagógicas de Giner y la Institución Libre de Enseñanza.

Existen otros casos en que obras de la moderna psicología científica, es decir, la psicología experimental servirán de un puente hacia la positivización de la filosofía. Por ejemplo, el caso de Eusebio Ruiz Chamarro. Ruiz Chamarro también fue alumno de Salmerón, y el autor de Psicología o ciencia del alma y también catedrático de Psicología del Instituto de Novicido de Madrid. Según Diego Nuñez Ruiz, Chamarro "pretende entroncar el Monismo krausista con el de la psicología esperimental» ${ }^{43}$. Finalmente, tenemos el caso de José Verdés Montenegro y Montoro ${ }^{44}$.

El Prof. José Verdés Montenegro y Montoro era socialista y profesor de psicología ${ }^{45}$. Verdés Montenegro y Montoro fue catedrático de psicología y lógica en Madrid, Orense y Alicante. Hay que relacionar pensamiento de Verdés Montenegro con las ideas de Salmerón, Adolfo G. Posada y Manuel Sales y Ferré y el replanteamiento de la actividad filosófica en el contexto científico-positivo y la formación en España de una línea de filosofía científica. Verdés Montenegro conocía a Urbano González Serrano y a Nicolás Salmerón. Según Doña M. ${ }^{a}$ Dolores Gómez Molleda, Verdés Montenegro y Montoro fue «discípulo predilecto de Salmerón" ${ }^{46}$. El Prof. Verdés Montenegro y Montoro también cobra importancia porque fue el traductor de la obra de Enrico Ferri, Socialismo y ciencia positiva (1895) ${ }^{47}$. Como nos dice Pedro Ribas, el libro de Ferri tuvo gran difusión en toda Europa y presenta un entroque entre el liberalismo spenceriano con el socialismo ${ }^{48}$.

\section{Conclusión}

Para resumir, lo que hemos querido hacer en esta ponencia es subrayar el interés que tuvo Unamuno por la psicología fisiológica dentro del contexto de su desarrollo intelectual y las oposiciones que 
hizo durante su regreso a Bilbao (1884 -1891). O sea, antes de ganar la Cátedra de Griego de la Universidad de Salamanca. Lo que hemos querido enfatizar es que el joven Unamuno fue uno de los primeros españoles que llegó a conocer las corrientes mas avanzadas de la psicología moderna, es decir, la psicología fisiológica de su tiempo. Es menester, situar este interés por la psicología dentro de la historia de la psicología dentro de España. Que yo sepa, las obras de historia de la psicología española, hasta la fecha, no han mencionado el papel de Unamuno en el desarrollo de la psicología moderna. También hemos querido indicar que aunque Unamuno tuvo contacto con los krausistas, los seguidores del krausismo y conoció bien el positivismo, su interés por la psicología fisiológica no brotó del krausopositivismo sino de un interés independiente. Aún hay mucho que hacer en cuanto a este tema. Hay que trabajar mucho más en la Casa Museo -ahora ya informatizada- para explorar e indagar sobre la comparación entre Unamuno y el pensamiento de González Serrano ${ }^{49}$. Otra senda que nos gustaría seguir en el futuro es la relación de este interés sobre la psicología físiológica de Unamuno y las obras y el pensamiento de Santiago Ramón y Cajal (1854-1934) ${ }^{50}$, Nicolas Achúcarro Lund (18801918) ${ }^{51}$ y Ramón Turró i Durdar (1854-1926) ${ }^{52}$.

\section{Notas} 9: 817.

1 Unamuno, Carta a Urales, "Sobre influencias extranjeras en mi obra". $O C E$,

2 UnAmuno, «En mi viejo cuartow. OC, 10:194.

3 Para una introducción breve sobre "historia de las mentalidades", ver M. TUŃón DE LARA, iPor qué la historia? (1981): pp 36-37 y para un ejemplo de esto aplicado al pensamiento español, ver D. NUÑEz RUIZ, La Mentalidad positiva en España: desarrollo y crisis. (Madrid, Tucar, 1975) y ABELLÁN, José Luis, Historia critica del pensamiento español. Tomo V(1): «La crisis contemporánea (1875-1936)». (Madrid 1989).

4 Ver nuestro estudio, «El joven Unamuno: Los años madrileños (1880-1884)». Anuario del Departamento de Filosofia. II, Universidad Autónoma de Madrid. Curso 1985/1986, pp 29-40.

5 Hemos estudiado esta época a fondo en nuestra tesis doctoral, ver "The Young Unamuno..." (1983), Capítulo IV, "The Bilbao Years". 47.

6 Emilio SalCEDo, Vida de Don Miguel. (Ediciones Anaya, Salamanca, 1970):

7 UnAMUNo, «Mi mirador». OC, 10:199.

8 UnAMUNo, «Sobre la erudición». OC, 3: 914.

9 UNAMUNo, «En mi viejo cuarto». $O C, 10: 194$.

10 Ver Unamuno, «Carta a Federico Urales. Sobre influencias extrangeras en mi obraw. OCE, 11: 817 . 


\section{Unamuno y la psicología moderna}

11 Ver nuestra bibliografia al final de este trabajo.

12 UNAMUNO, «En mi viejo cuarto». OC, 10:195.

13 UNAMUNO, «En mi viejo cuarto». OC, 10:195.

14 Cardinal Mercier, A Manual of Modern Scholastic Philosophy. (Routledge and Kagen Paul, London, 1960), Vol. 1.

15 Ver Ceferino González, Filosofía elemental, (Madrid. A. Pérez Dubrull 1884): 343 ss. Ver también G. Fraile, Historia de la filosofía española (B. A. C., Madrid, 1971).

16 Ver Ramón CARNICER, Entre la ciencia y la magia Mariano Cubi. (Editorial Seix Barral, Barcelona, 1969), y Luis GRANJEL, La frenología en España. (Universidad de Salamanca, Salamanca, 1973).

17 Sobre SIMARRo ver LOPEZ PIÑERo et al., Diccionario histórico de la ciencia moderna en España. Vol. 2 (1983), 327-330. Los únicos trabajos serios sobre Simarro son los de Temma KAPLAN, «Luis Simarro and the Development of Science and Politics in Spain 1863-1917», tesis doctoral aún inedíta de la Universidad de Harvard (1969). KAPLAN publicó algo de su tesis en las actas del III Congreso Nacional de Historia de la Medicina, "Luis Simarro, Spanish Histologist». Volume 2 (Valencia 1971), 523-533, 545-555. Sobre Mata ver, T. CARreras Artau,. Médicos, Filósofos (C.S.I.C., Barcelona, 1952), 63-77 y LÓPEZ PIÑERO et al., Diccionario Histórico, Vol. 2: 42-43.

18 Ver Gómez Molleda, Unamuno "agitador de espiritus" y Giner. Correspondencia inédita. (Narcea, Madrid, 1977). Ver además, de la misma autora, El socialismo español y los intelectuales... (Ediciones de la Universidad de Salamanca, Salamanca, 1980), sobre González Serrano: 56, 59, 200, 212, 298, 327, 331, 333 y 359.

19 Ver UnAmuno, «Programa de psicología, lógica y ética», (1886) manuscrito. Casa Museo Unamuno. Salamanca. Sobre esta época de Unamuno, ver SAlcedo, Vida de Don Miguel (1970): 50 y A. Lacy, Miguel de Unamuno. The Rhetoric of Existence. (Mouton, The Haque, 1967): 63-64.

20 Bain fue filosófo inglés de la escuela experimental. Fue profesor de Lógica y sus obras más importantes son The Senses and The Intellect (1855) y The Emotions and The Will (1859). Sobre Bain y Mill ver G. MURPHY, Historical Indtroduction to Modern Phychology (1949):102-108. De BAIN, Unamuno había leído, Logique deductive et inductive. Trad. G. Compayre. 2 vols. (Baillière, París, 1881) y se puede consultar en la Casa Museo. De John Stuart Mill, Unamuno conocía, Considerations on Representative Governament. (Parker, London, 1861), System of Logic, Ratiocinative and Inductive. 2 vols. (London. Longsman, Green, Reader and Dyer, 1872) y L' Utilitarisme. (Baillière, París, 1883). Todas estas obras se encuentran en la Casa Museo.

21 UNAMUNO, "Principales influencias extranjeras en mi obra». OCE, 9 (1971): 817.

22 UnAmUNO, «Sobre la erudición y la crítica». OC. 3: 914.

23 Sobre los krauso-positivistas ver D. NUÑEZ, "El Krausismo Positivo" en La mentalidad positiva en España: 77-109; J. L. ABELLÁ, Historia crítica del pensamiento español. (Madrid, 1989), Tomo V (1): «El Krauso-Positivismo, Filosofia Institucionista»: 108-120 y A. Jiménez GARCfA, El Krausimo y la Institucion Libre de Enseñaza. (Ed. Cincel, Madrid, 1987): "La evolución del krausismo: el krausopositivismo": 112-130. Sobre la historia de la psicología en España ver, los estudios de H. CARPINTERO, "The Historical Development of Spanish Psychology» Internet Web Sit: psd.fe4C@sis.ucm.es (August 1995); «La psicología de España: Pasado, Presente y Futuro». Revista de Historia 
de la Psicología, 1(1980): 33-58 y «The Introduction of Scientific Psychology in Spain» en Woodward, W and M. Ash (eds.), The Problematic Science in Nineteenth Century Thought. Praeger, New York, 1982: 255-275.

24 Ver NUNEEZ (1975): 92-93. GINER DE LOS RIOS, F., Lecciones sumarias de Psicología, 2." ed. (1878) en O.C. IV (Madrid, 1920 ).

25 Wilhlem Max Wundt (1832-1920), Gustav Theoder Fechner (1801-1887) y Hermann Helmholtz (1821-1994) fueron muy importantes para el desarrollo de la psicología experimental y la psicofisica. Sobre Wundt, ver D. ROBINSON, "Wilhelm Wundt" en Toward a Science of Human Nature. (Columbia University Press, New York, 1982):173175.

26 Ver G. SAnchez-Granjel-Santander, Pedro Dorado Montero, un penalista salmantino. (Junta de Castilla y León, Salamanca, 1990):13. Ver también, J. J. GIL CREMADES, El reformismo español. (Ariel, Barcelona, 1969): 267.

27 RiBor también publicó La Psicologie allemande (1879) que es una introducción a las obras de Wundt, Fechner y Helmholtz.

28 Hemos desarrollado esto antes en «Lección: Nicolás Salmerón y el krausopositivismo». Curso «NICOLÁs SALMERON Y SU TIEMPO». Cursos de Verano de la Universidad Complutense de Madrid. Aguadulce, Roquetas de Mar (Almería). Agosto 1992. Trabajo inédito.

29 Sobre Draper, ver la excelente introducción a la presentación de DIEGo NUNEZ, Draper, Historia de los conflictos entre la religión y la ciencia. (Ed. Alta Fulla, Barcelona, 1987): 7-39.

30 Ver La polémica de la ciencia española. Edición de Ernesto y Enrique GaRcta Camarero. (Alianza, Madrid, 1970) y José SALA CATALA, Ideología y ciencia biológica en España entre 1860 y 1881. La difusión de un paradigma. (CSIC, Madrid, 1987): 118-120.

31 Sobre estas polémicas hay que ver, José SALA CATALA, Ideología y ciencia biológica en España entre 1860 y 1881. La difusión de un paradigma. (CSIC, Madrid, 1987): 90. Ver la respuesta tradicional de la Iglesia a la obra de Draper, en las obras de J. M. ORTI y LARA, La ciencia y la Divina Revelación; Tomás CÁmARA, Contestación a la Historia del conflicto entre la religión y la ciencia de J. G. Draper (Valladolid, 1883); Miguel MiR, Armonía entre la ciencia y la fe (1881); A. CoMELLAS, Demostración de la armonta entre la Religión Católica y la ciencia; José MENDIVE, La Religión católica vindicada de las imposturas racionalistas (Madrid, 1883, 1887, 1897).

32 Nosotros hemos consultado la siguiente edicion: J. W. DRAPER, History of The Conflict between Religion and Science. (D. Appleton and Company, New York, 1876).

33 Sobre las ediciones y traducciones de la obra de Draper ver, D. Fleming, John William Draper and The Religion of Science. (University of Pennsylvannia Press, Philadelphia, 1950) y James Moore, The Post-Darwinian Controversies. (Cambridge, Cambridge University Press, 1981): 1949.

34 G. TIBERGHIEN, Ensayo teórico e histórico sobre la generación de los concimientos humanos. (Imp. de F. E. Centeno, Madrid, 1875). Trad. de A. García Moreno. "Apéndice» y notas por N. Salmerón y U. González Serrano.

35 SALMERON, «Prólogo» a Hermenegildo GINER DE LOS RIOS, Filosofía y arte. (Madrid, 1878): XII-XIII.

36 HAECKEL, E., Morfología general de los organismos. (Ed. Barris y Cia., Barcelona, 1887). Traducción de Sanpere y Miquel y revisión del Dr. Gaspar Sintiñon. 


\section{Unamuno y la psicología moderna}

37 SAlMERón, «Prólogo» a Hermenegildo GINER DE LOS Rfos, Filosofía y arte. (Madrid, 1878): XII-XIII.

38 SAlMERÓN, "Prólogo» a la traducción de Augusto T. Arcimis, de la obra de DRAPER, Historia de los Conflictos entre La Religión y La Ciencia. (Madrid, 1876), p. XVII.

39 NUÑEz (1975): Cap. VI «La difusión del naturalismo alemán»: 187 ss.

40 Discípulo de Salmerón y profesor de Psicología en la Institución Libre de Enseñanza. Ver NUÑEZ (1975): 212.

41 Valenti CAMP, Santiago, «Urbano González Serrano» Ideólogos, Teorizantes y Videntes. (Ed. Minerva, Barcelona, 1922 ?): 274.

42 Entre las más importantes obras de Gonzalez Serrano, tenemos Estudios sobre los principios de la moral en relación con la doctrina positiva. (Madrid, 1871), «Estudios sobre el positivismo*. Revista de Sevilla, V (1873), Estudios de Moral y Filosofia. (Madrid, 1875), Manual de psicología, lógica y ética. (Madrid, 1880-1887), La sociologia cientifica (1884) y La psicología fisiológica. (1886).

43 NUNEZ (1975): 211-212.

44 NUÑEZ (1975): 214.

45 Ver NUÑEZ (1975) y J. L. ABELLÁ (1989).

46 GÓMEZ MOLLEDA, M. D., El socialismo español y los intelectuales (Salamanca, 1980): 45.

47 NUNEZ (1975): 190-191.

48 Pedro RIBAS, «Unamuno socialista» en UnAmUNo. Escritos socialistas (Ed. Ayuso, Madrid, 1976): 37.

49 En la Casa Museo hay varias obras de GonZÁlez SERRANo entre ellas: Psicología del amor (F. Fe, Madrid, 1897); Preocupaciones sociales (Rodíguez Serra, Madrid, 1899) y otras, ver VALDÉS: 103.

50 En la Casa Museo se encuentran las siguientes obras de RAMÓN y CAJAL: Reglas y consejos sobre investigación biológica. (Moya, Madrid, 1913, 3." ed.; 4." ed. 1916; Recuerdos de mi niñez. 2 vols. (Moya, Madrid, 1917).

51 AchUCARRo fue alumno de Unamuno en Bilbao, ver SAlCEdo, Vida de Don Miguel: 48.

52 De TURRO, Unamuno conocía, Les origines de la connaissance. (Alcan, París, 1914); La base trófica de la inteligencia. (Residencia de Estudiantes, Madrid, 1918) y Filosofia crítica. (Ed. Catalana, Barcelona, 1918). También existe una correspondencia entre Unamuno y Turró en la Casa Museo.

\section{Bibliografía}

\section{Unamuno y la psicología moderna}

ABELlÁN, José Luis: Historia critica del pensamiento español. Tomo V (1): «La crisis contemporánea (1875-1936)», Madrid, 1989.

CACHO VIU,V.: La Institución Libre de Enseñaza. Rialp, Madrid, 1962.

CASTILLEJo, José: Wars of Ideas, 1937.

- Guerra de ideas en España, Madrid, 1976. 
ChABRAN-[GONZALEZ], [H.] Rafael: «Umamuno y la crítica de la razón positivista». Simposium sobre Miguel de Unamuno. Universidad del País Vasco. Vitoria, Alava, 1980. Ponencia inédita.

- The Young Unamuno: His Intellectual Development in Positivism and Darwinism (1880-1894)». Tesis doctoral aún inédita. Universidad de California, San Diego-La Jolla, 1983. Un resumen de esta tesis se ha publicado en Dissertation Abstracts International, Vol. 44, N. 7 (enero, 1984), p. 2158A.

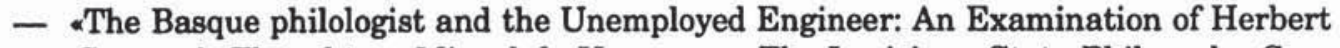
Spencer's Thought on Miguel de Unamuno*. The Louisiana State Philosophy Conference. Louisiana State University, Baton Rouge, Louisiana, 1983. Ponencia inédita.

- «Unamuno and Darwin». The Louisiana Conference of Hispanic Languages and Literature. Louisiana State University, Baton Rouge, Louisiana, 1984. Ponencia inédita.

- «Unamuno como opositor fracasado: una confrontación de paradigmas». Simposio sobre la Historia de la Ciencia. Instituto de Investigaciones Históricas. UNAM. Universidad Nacional Autónoma de México, México D.F., 1984. Ponencia inédita.

- «El joven Unamuno: Los años madrileños (1880-1884)». Anuario del Departamento de Filosofia II. Universidad Autónoma de Madrid. Curso 1985/1986: 29-40.

- «Miguel de Unamuno: Translator of Herbert Spencer . American Association of the Teachers of Spanish and Portuguese, Los Angeles, California, 1987. Ponencia inédita.

- «Unamuno's Early Salamanca Years». Revista Canadiense de Estudios Hipánicos. $11 / 2$ (Invierno 1987), 243-256.

- "Miguel de Unamuno: Traductor de Spencer . Anuario del Departamento de Filosofiá. Universidad Autónoma de Madrid. Curso 1986-87 y 1987-88, 33-42.

- «l joven Unamuno y su desarrollo intelectual». Ponencia inédita. Primeras Jornadas Unamunianas. Universidad de Salamanca. (Octubre 1991).

- «Lección: Nicolas Salmerón y el krausopositivismo*. Curso "NiCOLÁS SALMERON Y SUTIEMPO». Cursos de Verano de la Universidad Complutense de Madrid, Aguadulce, Roquetas de Mar (Almería). Agosto, 1992.

- -El Joven Unamuno (1891-1894): Centenario de su llegada a Salamanca (18911991)». Artículo manuscrito presentado para publicación en los Cuadernos de la Catedra Miguel de Unamuno. Septiembre, 1993.

DiAz, Elías: La filosofia social del krausismo, Madrid, 1973.

DRAPER, John William: History of the Conflicts Between Religion and Science. D. Appleton and Company, New York, 1875,1876, 1889. The International Scientific Series.

- [Draper, Juan Guillermo]. Historia de los Conflictos entre La Religión y La Ciencia. Prólogo de N. Salmerón a la traducción de Augusto T. Arcimís. (Madrid, 1876, 1." ed.; 2." ed. Ricardo Fe, Madrid, 1885; Alta Fulla Mundo Cientifico, Barcelona, 1987). Presentación de Diego Nuñez Ruiz.

Fernandez LaRrain, Sergio: Cartas inéditas de Unamuno. Rodas, Madrid, 1972.

Fleming. D: John William Draper and the Religion of Science. Philadelphia, 1950.

Gil Cremades, Juan José: El reformismo español. Ediciones Ariel, Barcelona, 1969.

- Krausistas y Liberales. Dossat, Madrid, 1981.

GINER DE LOS RfOS, Francisco: «Salmerón» Homenaje a la buena memoria de D. Nicolás Salmerón*. Trabajos filosoficos y discursos politicos. Imprenta de la Gaceta Administrativa, Madrid, 1911. 


\section{Unamuno y la psicología moderna}

GINER DE LOS Rfos, Hermenegildo: Filosofiá y arte. Imp. Minuesa de los Ríos, Madrid, 1878.

Gómez Molleda, M. ${ }^{a}$ Dolores: Los reformadores de la España contemporánea. CSIC, Madrid, 1966.

- El socialismo español y los intelectuales. Salamanca, 1980.

GONZAlez SERRANo, Urbano: Estudios sobre los principios de la moral en relación con la doctrina positiva. Madrid, 1871.

- «Estudios sobre el positivismo.». Revista de Sevilla, V,1873.

- Estudios de Moral y Filosofia. Librerías de Francisco Iravedra (Antonio Novo), Madrid, 1875. Prólogo de Manuel de la Revilla. Dedicación a Nicolás Salmerón.

- Manual de psicologia, logica y ética. Madrid, 1880-1887.

- Preocupaciones sociales, 1882.

— «La psicología novísima». Revista de España. 98 (1884): 404-426; 99 (1884): 33-50; 100 (1884): 47-71, 503-535.

- La sociología cientifica, 1884.

- La psicología fisiologica, 1886.

- «Nicolás Salmerón. Estudio crítico biográfico», en tomo IV de Celebridades españoles contemporáneos. Librería Fernando Fe, Madrid, 1903; París, 1903.

Heredia Soriano, A.: Nicolas Salmerón. Vida, obra y pensamiento. Tesis inédita. Universidad de Salamanca, 1972.

- "Nicolás Salmerón: Base bibliográfica para su estudio con cartas inéditas». Cuadernos Salmantinos de Filosofía, IX, 1982.

Jiménez GaRcta, Antonio: «Urbano González Serrano y la evolución de la filosofia krausista en el último tercio del siglo XIX (1868-1904)». Univeridad Complutense, Madrid, 1975.

- El Krausismo y la Institución Libre de Enseñanza. Madrid, 1986.

Jiménez LANDI, Antonio: La Institución Libre de Enseñanza. Tecnos, Madrid, 1973.

- Científicos de la Institución Libre de Enseñanza* en vv. En el Centenario de la Institución Libre de Enseñanza, 1977, 89-101.

LOPEZ MORILlAS, Juan; El Krausismo español. Fondo de Cultura Económica, México, 1965.

- The Krausist Movement and Ideological Change in Spain, 1857-1874. Cambridge University Press, Cambridge, 1981.

NUNEZ RuIZ, Diego: La mentalidad positiva en España: desarrollo y crisis. Tucar, Madrid, 1975.

NunEz, Diego y RiBas, Pedro: Unamuno. Politica y filosofia: artículos recuperados (1886-1924). Fundación Banco Exterior, Madrid, 1992.

Pérez GonzAlez, Fernando Tomás: La introducción del Darwinismo en la Extremadura decimonónica. Cáceres, 1987.

PosadA, Adolfo, G.: «Los fundamentales psicológicos de la Educación...* Boletín de la Institución Libre de Enseñanza [BILE], 358 (15 de enero 1892).

Robles, Laureano. Miguel de Unamuno. Epistolario inédito. 2 vols. Espasa Calpe, Madrid, 1991. Edición de L. Robles.

RIBAS, Pedro: «Unamuno socialista» en Unamuno. Escritos socialistas. Editorial Ayuso, Madrid, 1976.

Sala CatalÁ, José: Ideología y ciencia biológica en España entre 1860 y 1881. La difusión de un paradigma. CSIC, Madrid, 1987. 
SAlCEDo, Emilio: Vida de Don Miguel. Anaya, Madrid, 1964:47, 2." edición, Salamanca, 1970.

SALMERÓN, Nicolás: Obras completas. 4 vols. Madrid, 1911.

- "Prólogo» a Estudios de Religión de Calderón Llanés, 1873.

- «Prólogo» a Juan Guillermo Draper. Historia de los Conflictos entre La Religión y La Ciencia. Traducción de Augusto T. Arcimís. Madrid, 1876, $1{ }^{a}{ }^{a}$ ed.; $2{ }^{a}{ }^{a}$ ed. Ricardo Fe, Madrid, 1885; Alta Fulla Mundo Cientifico, Barcelona, 1987). Presentación de Diego Nuñez.

- «Prólogo* a Filosofia y arte de Hermenegildo Giner de los Ríos. Madrid, 1878.

- La filosofía de la vida, 1902.

SalmerON, Nicolas y GonZAlez Serrano, Urbano: «Apéndice» en G. Tiberghien, Ensayo teórico e histórico sobre la generación de los conocimientos humanos. Madrid, 1875.

SAnchez-Granjel Santander, G.: Pedro Dorado Montero, un penalista salmantino. Salamanca, 1990.

SANZ y Escartin, Eduardo: Reseña del libro Psicología de Urbano González Serrano en Revista de España, 83, 1881: 392-410.

SPENCER, Herbert; «The Comparative Psychology of Man* Mind, January, 1876.

— «Psicología comparada del hombre». Revista Contemporánea. I, 1876:7-20.

- Primeros principios. Trad. by José Andrés Irueste Madrid: Biblioteca Perojo, 1879.

- Estudios politicos y sociales. Biblioteca Científico-Literaria, Sevilla, 1879.

- Essays: Scientific, Political and Speculative. Williams and Norgate, London, 1891, 3 vols. Casa Museo Unamuno.

\section{UNAMUNO, MIGUEL DE}

\section{A. Manuscritos}

"Programa de psicología, lógica, y etica», (April 1886). Casa Unamuno, Salamanca. «Expediente académico, personal y de oposiciones». Archivo de Educación y Cultura, Alcalá de Henares.

- (1) «Programa de Metafisica» (1888).

- (2) «Programa de Metafísica" (1980).

\section{B. Obras completas}

Unamuno, Miguel de: Obras completas (OC). 15 vols. Afrodisio Aguado, S.A., Madrid, 1958-1964.

- Obras completas (OCE). 9 vols. Edición de M. García Blanco. Escelicer, Madrid, 1967-1971.

\section{Correspondencia}

Cartas a Pedro Múgica en Cartas inéditas. Edición Fernández Larian. Rodas, Madrid, 1972.

Carta a Francisco Urales. Ver La evolución de la filosofía en España. Barcelona, 1934. 


\section{Unamuno y la psicología moderna}

- Carta a Pedro Múgica (29 de abril de 1890).

- Carta a Pedro Múgica (23 de noviembre de 1891).

- Carta a Pedro Múgica (5 de abril de 1892).

- Carta a Pedro Múgica (28 de mayo de 1893).

Robles, Laureano. Miguel de Unamuno: Epistolario inédito. 2 vols. Espasa Calpe, Madrid, 1991. Edición de L. Robles.

Urales, F.: La evolución de la filosofía en España. Laia, Barcelona, 1977.

VALDÉs, Mario: «El residuo spenceriano en Unamuno». Insula, 17 (1963): 32.

- y VAldÉs, M." E.: An Unamuno Sourcebook. University of Toronto, Toronto, 1973: xvii-xx

WiltshiRe, D.: The Social and Political Thought of Herbert Spencer. Oxford University Press, New York, 1978.

\section{Obras sobre Historia de la psicología en España}

CARPINTERo, H. «The Historical Development of Spanish Psychology». Internet Web Sit: psd.fe4@sis.ucm.es (August 1995).

- "La psicología de España: Pasado, Presente y Futurow. Revista de Historia de la Psicologíá, 1 (1980): 33-58.

- «The Introduction of Scientific Psychology in Spain* in

WoodWARD. W and M. ASH (eds.): The Problematic Science in Nineteenth Century Thought. Praeger, New York, 1982: 255-275.

LOPEZ PINERo, J. M. et. al.: Diccionario histórico de la ciencia moderna en España. Ed. Península, Barcelona, 1983. 2 vols.

\section{Obras sobre Historia de la psicología en general}

BoRING, Edwin G.: A History of Experimental Psychology. D. Appleton-Century Company, New York, 1929.

Freeman, G. L.: Physiological Psychology. Van Nostrand, New York, 1948.

Herrnstein, Richard J. and Edwin G. Boring (eds.): A Source Book on the History of Psychology. Harvard University Press, Cambridge, Mass, 1965.

Kendere, Howard H.: Historical Foundations of Modern Psychology. Brooks/Cole Publishing Company, Pacific Grove, 1987.

MURPHEY, Gardner: Historical Introduction to Modern Psychology. Harcourt, Brace and Company, New York, 1949.

RIEBER, R.W.: Wilhelm Wundt and the Making of a Scientific Psychology. Plenum Press, New York, 1980.

RoBINSON, D. Willhelm Wundt: Toward a Science of Human Nature. Columbia University Press, New York, 1982.

\section{Algunas obras sobre psicología en la Casa Museo Unamuno}

LOTZE, Hermann: Principes generaux de psychologie physiologique. Baillière, París, 1887.

Rıвот, T.: La psychologie allemande contemporaine. Baillière, París, 1879. 
- Les maladies de la memoir. Baillière, París, 1881.

- Psychologie de l'attention. Alcan, París, 1889.

- Les maladies de la volonte. Alcan, París, 1894.

RICHET, Charles: Essai de psychologie moderne. Alcan, París, 1887.

Siciliani, Pierre: Protegomenes a la psychologie moderne. Baillière, París, 1880.

SPENCER, Hernert: Principes de psychologie. Traducción de Th. Ribot y A. Espinas. Baillière, París, 1875,2 vols.

WunDT, W.: Grundzüge der physiologischen. Psychologie. 2 vols, Engelmann, Leipzig, 1893. 